\title{
Analysis of peptide PSY1 responding transcripts in the two Arabidopsis plant lines: wild type and psy1r receptor mutant
}

\author{
Khalid Mahmood ${ }^{1,2}$, Rubini Kannangara', Kirsten Jørgensen ${ }^{1}$ and Anja T Fuglsang ${ }^{1,2^{*}}$
}

\begin{abstract}
Background: Small-secreted peptides are emerging as important components in cell-cell communication during basic developmental stages of plant cell growth and development. Plant peptide containing sulfated tyrosine 1 (PSY1) has been reported to promote cell expansion and differentiation in the elongation zone of roots. PSY1 action is dependent on a receptor PSY1R that triggers a signaling cascade leading to cell elongation. However little is known about cellular functions and the components involved in PSY1-based signaling cascade.

Results: Differentially expressed genes were identified in a wild type plant line and in a psy $1 r$ receptor mutant line of Arabidopsis thaliana after treatment with PSY1. Seventy-seven genes were found to be responsive to the PSY1 peptide in wild type plants while 154 genes were responsive in the receptor mutant plants. PSY1 activates the transcripts of genes involved in cell wall modification. Gene enrichment analysis revealed that PSY1-responsive genes are involved in responses to stimuli, metabolic processes and biosynthetic processes. The significant enrichment terms of PSY1-responsive genes were higher in psy1r mutant plants compared to in wild type plants. Two parallel responses to PSY1 were identified, differing in their dependency on the PSY1R receptor. Promoter analysis of the differentially expressed genes identified a light regulatory motif in some of these.
\end{abstract}

Conclusion: PSY1-responsive genes are involved in cellular functions and stimuli responses suggesting a crosstalk between developmental cues and environmental stimuli. Possibly, two parallel responses to PSY1 exist. A motif involved in light regulation was identified in the promoter region of the differentially expressed genes. Reduced hypocotyl growth was observed in etiolated receptor mutant seedlings.

Keywords: Cellular functions, Gene enrichment analysis, Microarray, Signaling cascade, Small signaling peptides

\section{Background}

In the past few years, our understanding of signals required for cell-to-cell communication during plant development has increased tremendously. Identification of components that mediate signaling serves as a landmark in understanding the mechanism of cell-tocell communication in planta. Several components such as phytohormones, mobile transcription factors, mobile small RNAs and peptides serve this purpose $[1,2]$. Phytohormones are lipophilic compounds which are active at very low concentrations and involved in plant

\footnotetext{
*Correspondence: atf@plen.ku.dk

'Department of Plant and Environmental Sciences, University of Copenhagen, Copenhagen, Denmark

PUMPKIN, Centre for membrane pumps in cells and disease, Copenhagen, Denmark
}

(c) 2014 Mahmood et al.; licensee BioMed Central Ltd. This is an Open Access article distributed under the terms of the Creative Commons Attribution License (http://creativecommons.org/licenses/by/2.0), which permits unrestricted use, distribution, and reproduction in any medium, provided the original work is properly credited. The Creative Commons Public Domain Dedication waiver (http://creativecommons.org/publicdomain/zero/1.0/) applies to the data made available in this article, unless otherwise stated.

growth ranging from embryogenesis to senescence [2]. Similarly, small-secreted peptides are now emerging as growth regulators and many of them are involved in basic functions of cell growth and development. More than 1000 genes are annotated as encoding putatively secreted peptides in the Arabidopsis genome but very few are known to be involved in specific cellular signaling $[2,3]$. However, the precise role and mechanism proceeded by secreted peptides is yet to be established. Secreted peptides have now been recognized as a new class of intracellular signal molecules, which coordinate and specify cellular functions in plants. As a new class of intracellular signal molecules, the role of these secreted peptides could be explored further. 
Plant cells transduce signals utilizing surface receptors binding to ligands present in the apoplast [4]. The S-locus receptor $Z m P K 1$ from Maize was the first receptor kinase identified in plants [5], and many receptors have been identified in plants ever since [6,7]. Signaling molecules can elicit different signaling pathways, and a single receptor can also respond to more than one signal molecule [8]. Perception of signal molecules [9], and adjustability to different environmental conditions [10] are interesting characteristics of these signaling cascades. Plants demonstrate different growth patterns under different environmental conditions owing to asymmetric elongation or cell division [11-14]. They also exhibit flexibility in the size and numbers of produced organs to ensure diversity and specificity in perception of external stimuli $[1,2,10-15]$.

Plant peptide containing sulfated tyrosine (PSY1) is a tyrosine-sulfated peptide isolated from Arabidopsis cell suspension medium [16]. It promotes cell expansion and differentiation in the elongation zone of roots at nanomolar concentration. This 18 -amino acid glycopeptide is derived from a 75-amino acid precursor polypeptide containing an $\mathrm{N}$-terminal signal peptide [16]. PSY1 is believed to bind the extracellular domain of Leucine rich Repeat Receptor Like Kinase (LRR-LK), which is named as a receptor of PSY1 (PSY1R). PSY1 and its receptor PSY1R are expressed throughout the whole plant with higher expression in shoot apical meristem and elongation zone of roots. PSY1 is known to be highly up-regulated after wounding [16]. Exogenous application of purified PSY1 peptide to suspension cell culture induces cellular proliferation, expansion and elongation while overexpression of Arabidopsis PSY1 causes longer roots with larger cotyledon as compared to wild type [16]. Recently, receptor PSY1R and peptide PSY1 were found to be involved in plant defense $[17,18]$. The PSY1R might integrate growth promotion and defense signals leading to modulation of cellular plasticity, and may allow the cells to adjust towards environmental changes.

In order to understand the role of PSY1 and its receptor PSY1R, a full genome microarray study was performed. Identification of genes responding to PSY1 is a bottleneck in explaining the specific signaling phenomenon. This is the first comprehensive study to elucidate components of the PSY1-based signaling cascade using full genome microarray in response to exogenously applied PSY1. We found that several genes, involved in plethora of physiological functions, are differentially expressed after PSY1 exposure. Our study indicates that two PSY1 responses exist. The promoter analysis leads to identification of a light regulatory motif in differentially expressed genes of psy1r mutant plants.

\section{Results and discussion}

\section{Genome wide analysis of two plant lines after PSY1} treatment and validation of microarray data

In order to understand the PSY1-based signaling cascade, we decided to identify genes affected by PSY1. For this purpose, transcriptome analyses were performed on two plant lines after PSY1 treatment. The two plant lines (wild type and psy1r) were germinated and grown hydroponically for one week under sterile conditions. Then both lines were treated with PSY1 peptide $(10 \mathrm{nM})$ for 4 hrs before mRNA isolation for microarray analysis (Figure 1). Three independent biological samples were prepared and an Arabidopsis Gene Expression Microarray (V4) of one color was used.

In the transcriptome analysis, a number of genes were identified using the criteria; $\mathrm{P}<0.05$ and fold change $>2$ or $<-2$. Gene expression analysis after peptide treatment of wild type plants revealed significant differential expression of 77 genes. Among them 51 genes were up-regulated while 26 genes were down-regulated (Additional file 1: Table S1). Mutation of the receptor resulted in 154 genes with altered expression levels as compared to wild type (Figure 2). Among them, 102 genes were down-regulated while 52 genes were up-regulated in receptor knockout plants indicating the lack of a downstream response to the treatment (Additional file 2: Table S2). Interestingly, the number of differentially expressed genes (261) was higher when comparison was made between PSY1-treated wild type and PSY1-treated receptor knockout lines (psy1r) (Additional file 3: Table S3). Examination of Gene Ontology (GO) terms in both plant lines after PSY1 treatment demonstrated the specific contribution of different classes of biological functions in each category (Figure 2, Additional file 4: Table S4). Individual genes of each category were assigned to six different classes of relevant putative biological functions that were derived automatically using AmiGO. Interestingly all three comparisons showed the overall same distribution. Maximum numbers of differentially expressed genes were involved in cellular and biosynthetic processes while the major part of the genes were also involved in regulation and metabolism (Figure 2). The category "reproduction" was not found after PSY1 treatment in wild type while this category was present in receptor knockout plants (Figure 2). Additionally, the number of differentially expressed genes attributed as kinases or phosphatases were higher in receptor mutant plants as compared to PSY1 responsive genes (Additional file 1: Table S1 and Additional file 2: Table S2).

The expression levels found in the microarray study were confirmed by quantitative real time RT-PCR for selected genes (Figure 3). The genes were selected in view of their role in cell elongation with range of differential expression to validate microarray data. The selected 


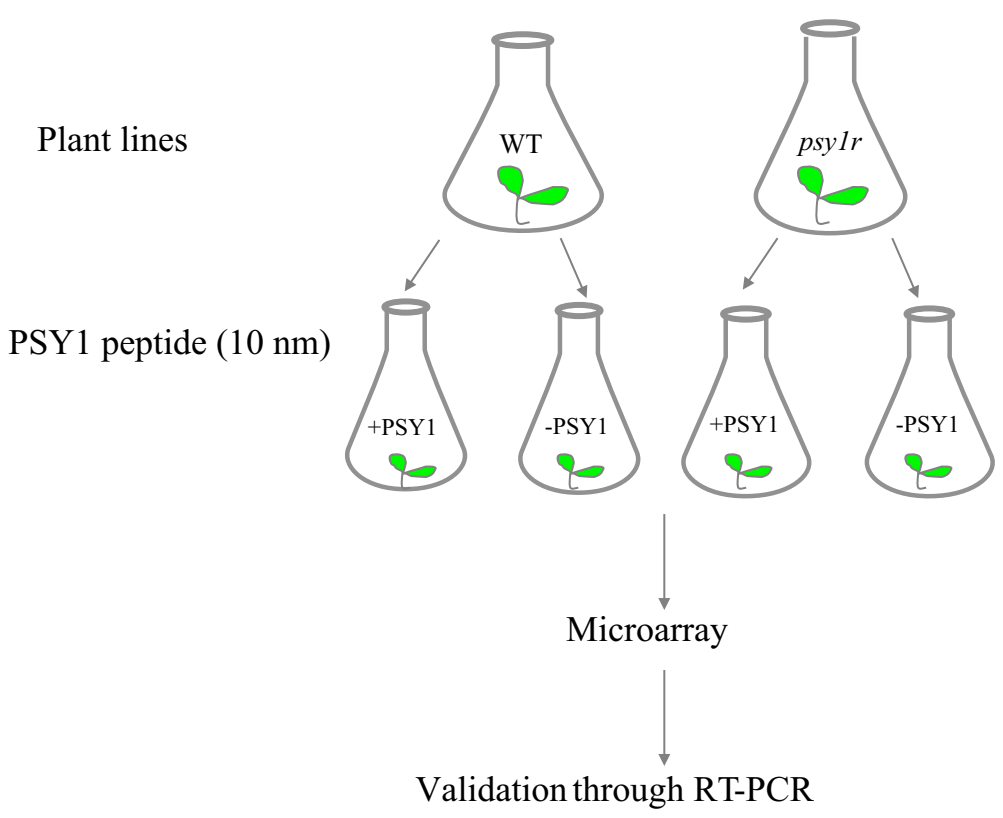

Figure 1 Schematic presentation of experimental set-up. Two plant lines were grown hydroponically for one week and following treated with $10 \mathrm{nM}$ PSY1 for $4 \mathrm{hr}$ before RNA extraction. Three independent biological samples were prepared.

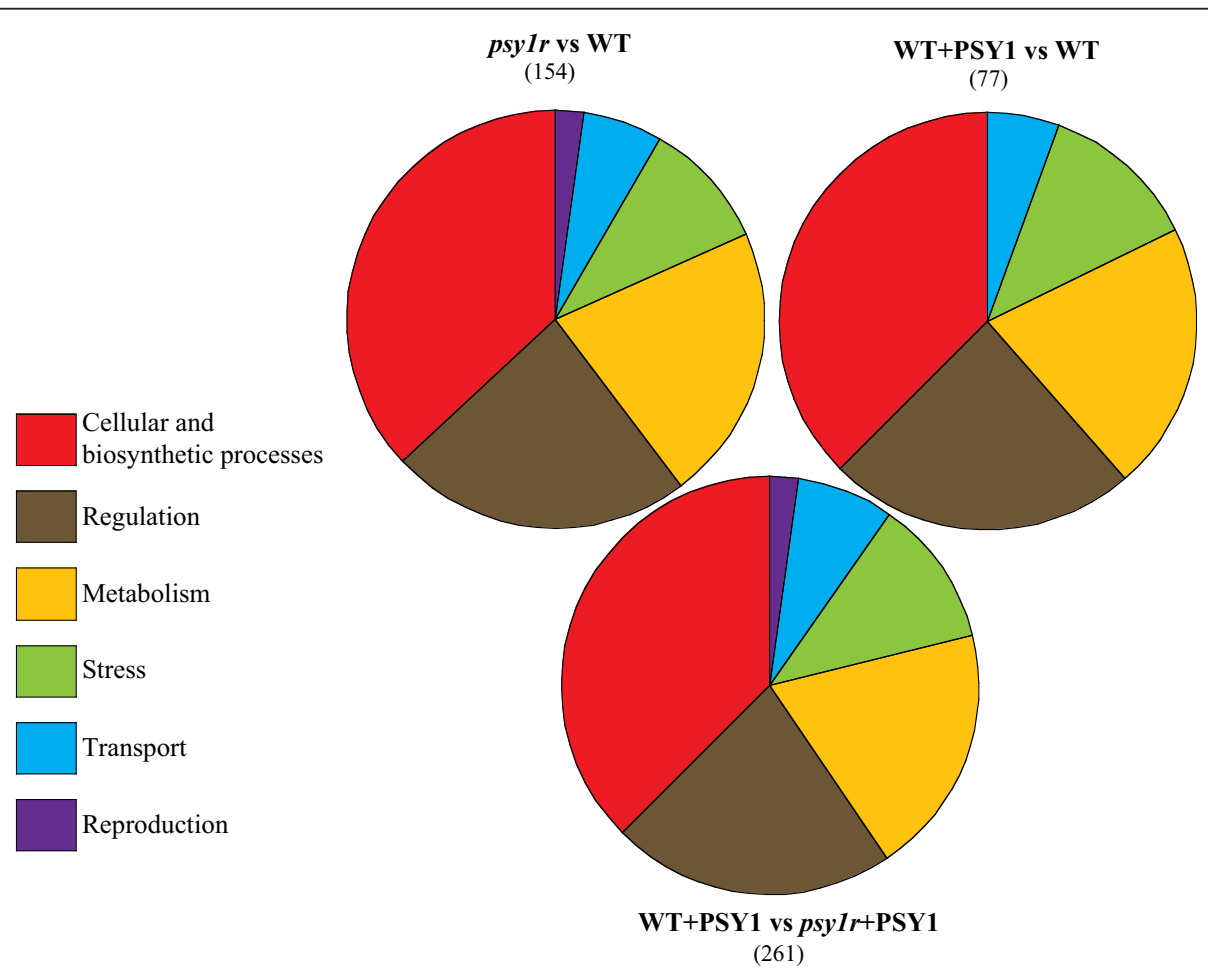

Figure 2 Gene Ontology (GO) terms depicting the distribution of expressed genes by Amigo GO (http://amigo.geneontology.org/cgi-bin/ amigo/go.cgi). Venn diagram represents the percentage of each biological function obtained by genes involved in corresponding functions divided by all expressed genes. The category psy $7 \mathrm{r}$ vs WT that represents the percentage of differentially expressed genes attributed to specific biological functions in psy1r mutant plants compared to wild type (psy1r vs WT). The category "WT + PSY1 vs WT" represents the percentage of differentially expressed genes after PSY1 treatment compared to untreated wild type. Other category "WT + PSY1 vs psy $1 r+$ PSY1" highlights the percentage of differentially expressed genes in PSY1-treated receptor mutant plants compared to PSY1-treated wild type plants. 


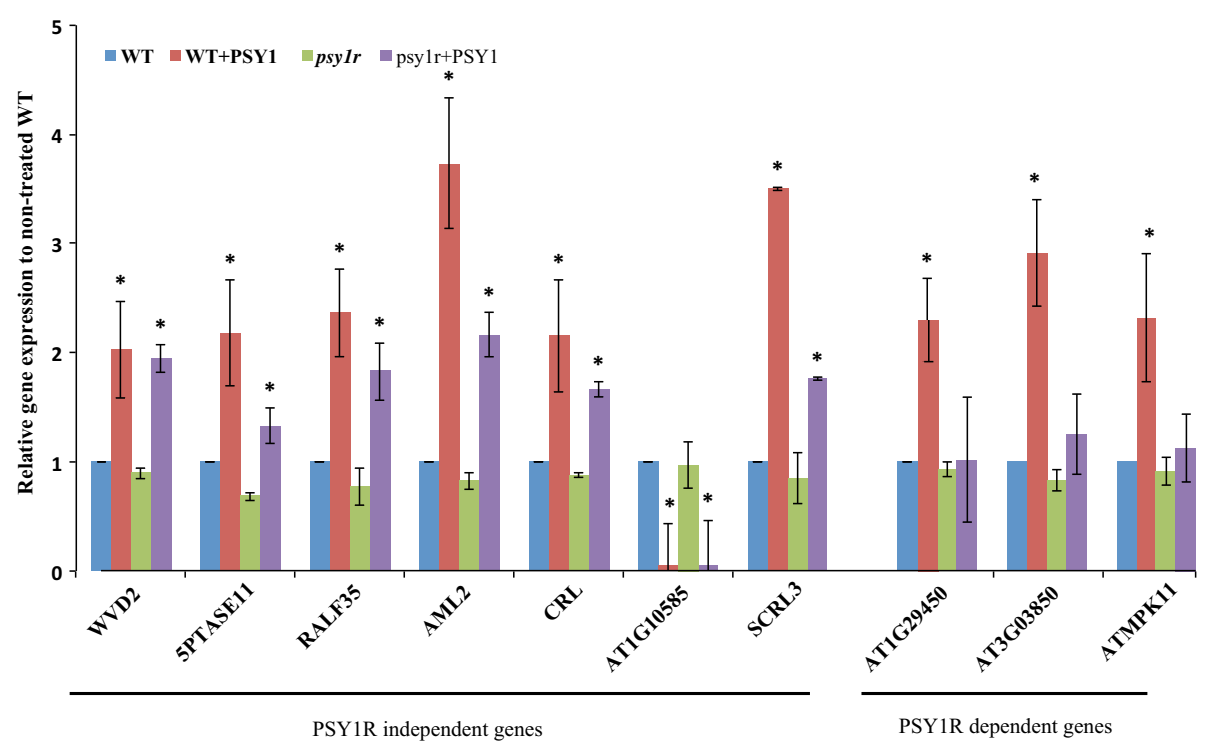

Figure 3 Validation of microarray data through qRT-PCR of selected genes. The transcript levels in plants were assessed following $4 \mathrm{~h}$ exposure of $10 \mathrm{nM}$ of PSY1 to one-week-old plants. Relative gene expression levels were compared with wild type control (defined value of 1). The relative transcript levels were calculated from three independent biological replicates. ${ }^{*}$ Indicates significance calculated among treatments using Tukey's test $(P<0.05)$.

genes included a novel protein WAVE-DAMPENED 2 (WVD2, AT5G28646) involved in cell expansion and root waving. Another gene that encodes a phosphatidylinositol polyphosphate 5-phosphatase (5PTase11, AT1G47510) also up-regulated after PSY1 treatment was selected, and so was RALF35, which is not characterized but belongs to another family of signaling peptide. Other tested proteins included AML2 (AT2G42890), which is involved in embryo development, CRL (AT5G51020), which is implicated in pattern of cell division and MPK11 (AT1G01560), which influences differentiation and plastid division. The specific primers used for each gene are listed in Additional file 5: Table S5. The qRT-PCR results generally agreed with the microarray data, however quantitative differences in expression levels were observed. In our experiments, the microarray was found to be more sensitive than the qRT-PCR data (Figure 3). Importantly, the qRT-PCR confirmed the presence of two groups of PSY1 responsive genes, one group dependent on the presence of PSY1R, the other group independent of PSY1R.

\section{Identification of enriched GO terms of differentially expressed genes in both plant lines}

In order to identify enriched GO terms, an analysis of over-representing GO categories using AgriGO (Fisher's exact test $<0.05$ ) was conducted [19]. Over-representing GO terms were divided into Biological Processes (BP), Cellular Component (CC) and Molecular Function (MF). According to BP, 32 categories were significantly enriched in wild type after PSY1 treatment. To make it more simple and visual, the over-representing GO categories were drawn manually. These over-representing GO categories include response to hormone stimulus, regulation of biosynthetic processes and regulation of transcription (Figure 4A). When looking at cellular components (CC), four significant enriched categories were observed with majority of genes localized to cell wall (Figure 4B). Cell wall loosening requires lowering of the apoplastic $\mathrm{pH}$ as well as structural changes such as breakage of loadbearing bonds through enzymes [20]. According to molecular functions, two over-represented GO categories were found namely transcription factor activity and carboxylesterase activity (Figure 4C). Esterases remove methyl groups from polysaccharides and can thereby cause breakage of polysaccharides [21]. This is suggested to be one of the mechanisms required for cell elongation [22-24], and would support a mechanism of PSY1-induced cell growth.

A functional enrichment study of differentially expressed genes in the psy1r mutant plants revealed that $52 \mathrm{GO}$ terms were significantly enriched. The most prominent enriched functions in BP were response to stimuli, regulation of transcription, metal ion transport, flower development and response to abscisic acid stimulus (ABA) (Figure 5A). Response to stimuli and regulation of transcription are believed to happen through perception of PSY1 peptide by the receptor PSY1R as extracellular signals mediate specific cellular functions by triggering a signaling cascade that result in modulation of transcription factor activity [25]. Other significant enriched terms are metal ion transport genes (AT5G26690, AT2G28160, AT3G46900, AT3 G48970 and AT5G52710) mainly involved in copper and 


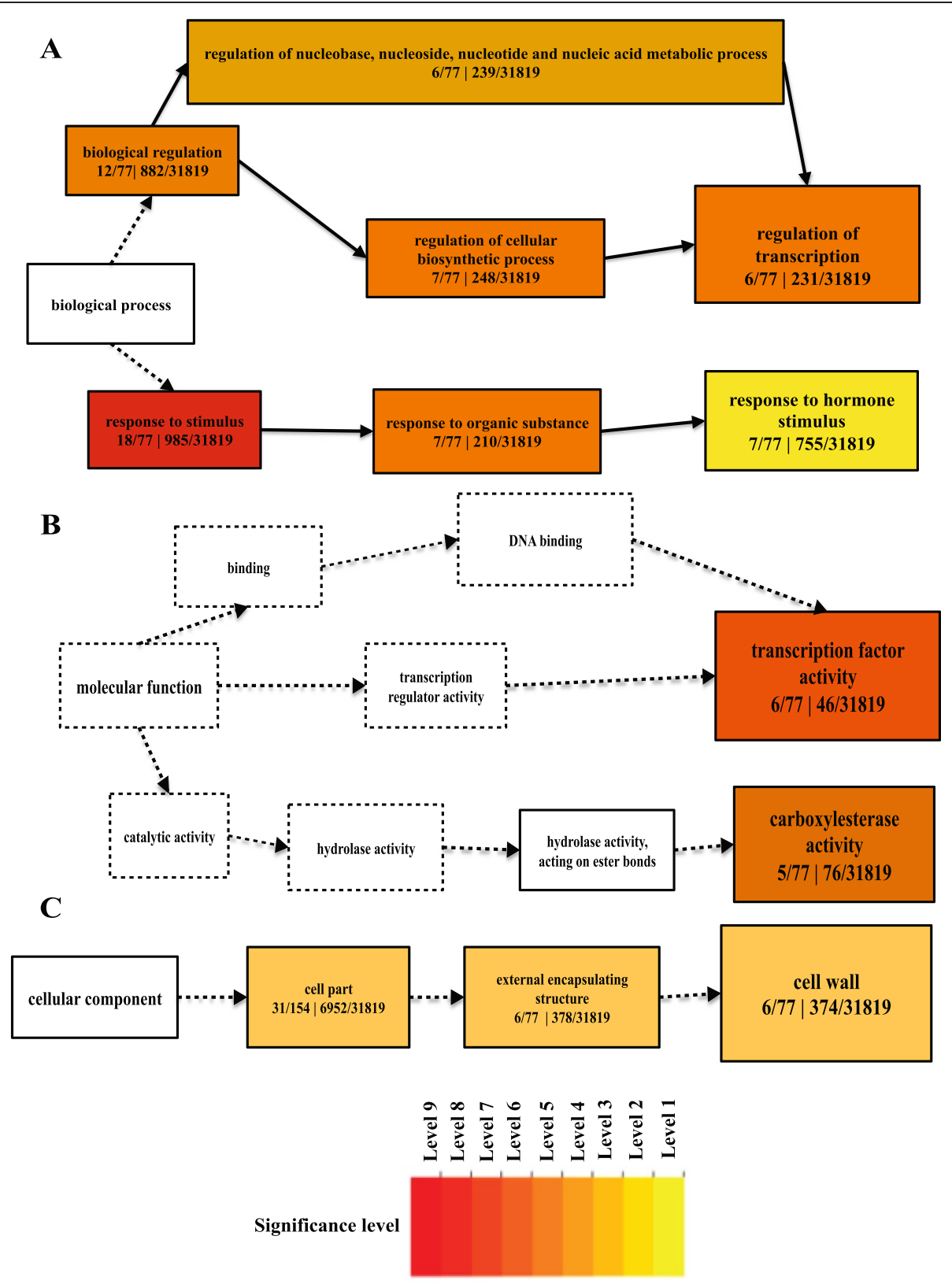

Figure 4 GO-terms enrichments of differentially expressed genes in wild type after peptide PSY1 treatment. The hierarchical graph of over-represented terms in Biological Processes (A), Molecular Function (B) and Cellular Component (C) by Singular Enrichment Analysis (SEA) using AgriGO. Boxes in the graph represent GO term labeled by their GO ID, term definition and statistical information. The significant term (adjusted $\mathrm{P}<0.05$ ) is marked with color while non-significant terms are shown as white boxes. The boxes contain GO term labeled by their definition and numbers represent differentially expressed genes of a category divided by known total number of genes involved in specific GO term. The color-coding of a box represents the significance level. Solid, dashed and dotted lines represent two, one and zero enrichment terms at both ends connected by line, respectively. The rank direction of graph runs from left to right.

iron transport. Both iron and copper transporters contribute to root elongation of Arabidopsis thaliana [26,27]. The metal ion transport GO term' is also found in the psy1r mutant plants. The GO term "response to abscisic acid stimulus" is enriched in psy1r mutant plants. Abscisic acid is involved in various processes including biotic and abiotic stress responses. A number of the differentially expressed genes (AT5G01540, AT3G28580, AT1G29395, AT1G73330 and AT1G48000) have previously been found to exhibit a negative regulation on abscisic acid response during growth [28]. Recently Mosher et al. (2013) found an involvement of PSY1R in plant defense [18]. This effect may be caused by changes in ABA levels, since ABA has profound roles in modulating diverse plant-pathogen interactions mediated 


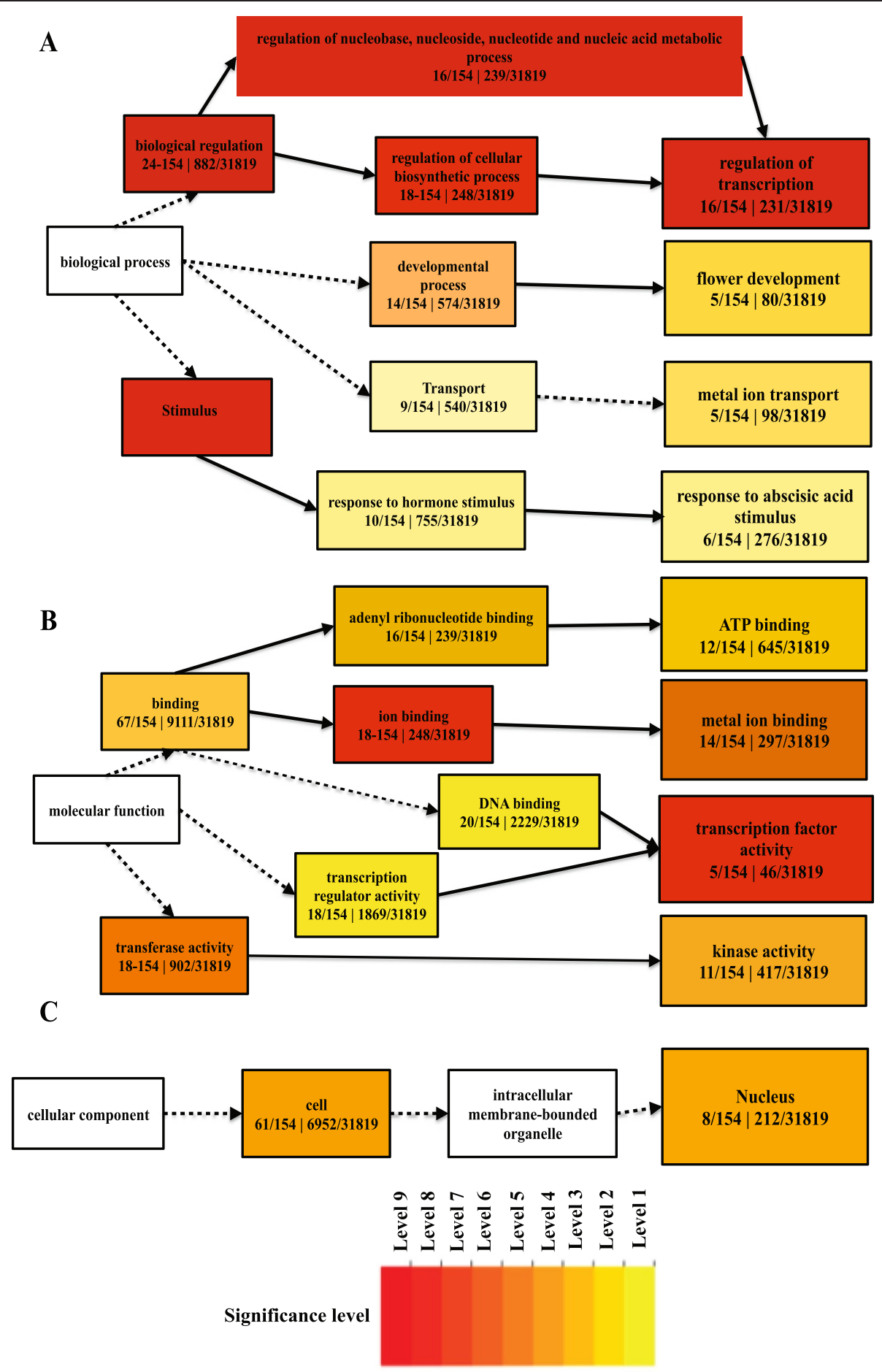

Figure 5 GO-terms enrichments of differentially expressed genes in receptor knockout (psy1r) plants. The hierarchical graph of over-represented terms in Biological Processes (A), Molecular Function (B) and Cellular Component (C) by Singular Enrichment Analysis (SEA) using AgriGO. Boxes in the graph represent GO term labeled by their GO ID, term definition and statistical information. The significant term (adjusted $\mathrm{P}<0.05$ ) is marked with color while non-significant terms are shown as white boxes. The boxes contain $\mathrm{GO}$ term labeled by their definition and numbers represent differentially expressed genes of a category divided by known total number of genes involved in specific GO term. The color-coding of a box represents the significance level. Solid, dashed and dotted lines represent two, one and zero enrichment terms at both ends connected by line, respectively while color line represents negative correlation to the enrichment level of term. The rank direction of graph runs from left to right. 
by cross talk with the jasmonic acid and salicylic acid signal pathways [29]. Genes found in the psy1r mutant plants involved in GO term "flower development" (AT4G16280, AT5G67060, AT1G24260, AT4G01500 and AT3G50330) are mainly involved in promotion of transition of vegetative meristem to reproductive development, carpel formation and ovule development [30-32]. According to cellular component $(\mathrm{CC})$, over-representing $\mathrm{GO}$ term found in the psy1r mutant plant is nucleus (Figure $5 \mathrm{~B}$ ). This is not surprising due to the increase in transcription factor activity occurring in the nucleus. According to molecular functions (MF), 22 categories were significantly enriched. The most significant enriched functions were ion binding followed by transcription factor activity, kinase activity and ATP binding (Figure 5C). These molecular function GO terms make sense in psy1r mutant plants due to PSY1R role in these functions and being a receptor of peptide PSY1.

In the PSY1-treated psy1r mutant plants, 62 GO terms were found according to biological component (BP) and these numbers were higher in the PSY1-treated psy1r mutant plants than in untreated psy1r mutant plants. GO terms lacking in the untreated psy1r mutant is "response to auxin stimulus" and "cellular amino acid metabolic processes". No differences were observed according to cellular component. When comparing molecular function GO terms of PSY1-treated and untreated psy1r plants, most prominent difference is the carboxylesterase activity observed in peptide-treated plants. This enzyme activity is completely absent in the untreated mutant plant line.
These differences encouraged a more comprehensive analysis of differentially expressed genes in the two plant lines.

\section{Receptor dependent and independent response to peptide PSY1}

In order to compare the affected genes Venn diagrams were drawn. The diagrams provide a comparison of differentially expressed genes in all three treatments (Figure 6A). The diagrams illustrate that some differentially expressed genes are common among treatments and some solely expressed in one treatment but absent in other treatments. Additionally a simplified comparison is made between peptide-treated wild type and peptide-treated psy1r mutant plants (Figure 6B). It revealed that PSY1 does trigger a response even in the absence of PSY1R, suggesting the existence of two parallel responses, named as a) PSY1R-independent response and b) PSY1R-dependent response. The genes that responded to PSY1 in both plant lines belongs to the PSY1Rindependent response, while the PSY1R dependent response consists of genes that are differentially expressed as response to PSY1 only in the presence of the receptor PSY1R. The PSY1R-receptor independent response includes 46 genes expressed in both plant lines after PSY1 treatment (Figure 6B). There could be two possible reasons for transcripts expression in psy1r-independent response. The first one might be due to absence of natural receptor PSY1R, PSY1 binds to a low affinity receptor that
A WT+PSY1 vs WT (77)

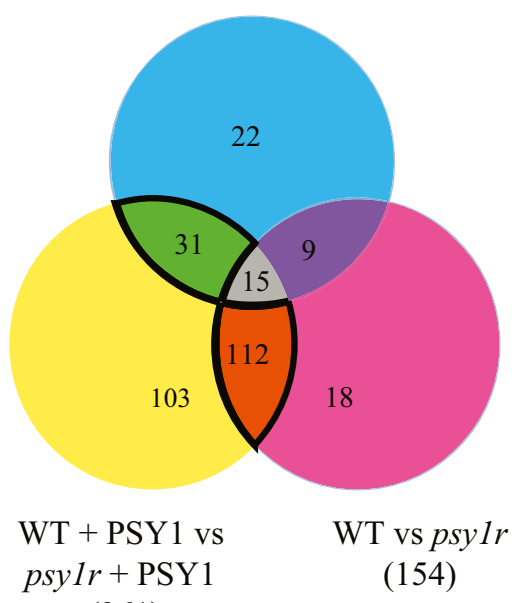

B WT+PSY1 vs WT (77)

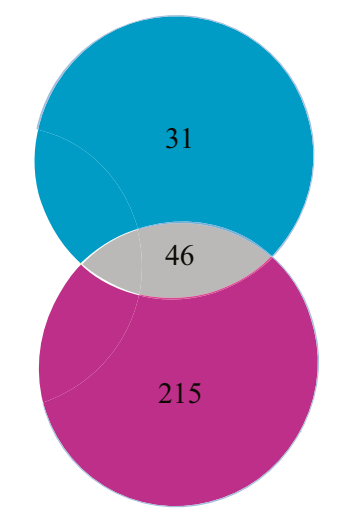

$\mathrm{WT}+\mathrm{PSY} 1$ vs psylr + PSY1

(261)

(261)

Figure 6 Comparison among differentially expressed genes in both plant lines with and without peptide treatment. A) Venn diagram comparing differentially expressed genes in all three treatments. Genes marked "Bright green" are common between both plant lines after PSY1 treatment, genes marked "gray" are differentially expressed in all three treatments. Genes marked with "violet color" are common between peptide-treated wild type and untreated psy1r mutant plants. Genes marked with "yellow", "blue color" and "pink color" are solely expressed in one treatment but absent in the other treatments. The 112 genes marked with "orange" are common between treated and untreated psy $1 r$ mutant plants. B) Venn diagram of PSY1-responsive genes. Grey: genes differentially expressed in both plant lines after PSY1 treatment, blue and pink represents genes induced in wild type and in psy1r mutant plants, respectively, after PSY1 exposure. 
capable of activating another signaling cascade results in transcripts expression. Alternatively, second one may explain that the addition of exogenous PSY1 peptide in the presence of endogenous PSY1 may cause homeostatic disturbance and a parallel signaling cascade is initiated. Nevertheless, a clear response is observed after exogenous peptide treatment.

Interestingly, when analyzing the over-represented GO categories it is found that the PSY1R-independent pathway represents the cell wall modifications (hydrolase activity), while the PSY1R-dependent pathway represents the response to stimuli, regulation of transcription, metal ion transport, flower development and response to abscisic acid stimulus.

\section{Identification of light-responsive cis elements in promoters of differentially expressed genes}

A search for common elements (cis elements) in the PSY1 affected genes was carried out using the Promomer tools in the Botany Array Resource [33]. The Promomer tools use alignment of sequences of genes and enumerative method to find motifs. We searched for over-represented 6-bp motifs in the 1-kb upstream promoter region (Table 1). The data indicated that all putative motifs are TA rich. It is known that TA rich sequences in the core promoter region act in promoting or repressing genes at the transcriptional level [34]. The highly significant motifs include TATATA and TGTATA (Table 1), which are a part of the light regulatory motif (TGTATATAT). The TGTATATAT motif was previously shown to be involved in the network of light regulated genes [35]. This motif is found in the promoter region of 14 genes of psy1r mutant plants and in only two PSY1-responsive gene promoters in wild type plants, accounting for $9 \%$ and $2 \%$ of all differentially expressed genes, respectively (Table 1). The fourteen genes of psy1r mutant plants containing the light regulatory motif in the promoter region are: Isochorismate synthase 2 (ICS2, AT1G18870), Knox Arabidopsis thaliana meinox (KNATM, AT1G14760), RXW8 (AT1G58520), AT1G23205, Ovate family protein 16 (OFP16, AT2G32100), AT3G62990, Cytochrome P450 (CYP82C2, AT4G31970), NGA4 (AT4G01500), Peroxidase 52 (PRX52, AT5G05340), Sweet 12 (AT5G23660), AT5G46080, NDR1-like 3 (AT5G06320), Longifolia 1 (AT5G15580) and AT5G21910. The Longifolia 1 is known to regulate longitudinal cell elongation in Arabidopsis thaliana [36]. The finding of TGTATATAT in differentially expressed genes of psy1r mutant plants may suggest an involvement of PSY1R in light response. A separate experiment was conducted in order to test if psy1r knockout plants react differently to the lack of light. Elongation of hypocotyls of etiolated psy1r plants was analyzed after 5 days of growth. Comparison of hypocotyls of darkgrown plants revealed shorter hypocotyls in psy1r mutant plants compared to wild type (Figure 7). These results also support the view that PSY1R contributes to hypocotyl cell elongation in response to light.

\section{Conclusions}

Small signaling peptides perceived by receptors are situated in specific cells to control growth and development by eliciting a vast array of physiological responses. PSY1 is a secreted peptide and its action is dependent on a receptor PSY1R that triggers a signaling cascade leading to cell elongation. However, the targets of this signaling pathway are yet to be studied in detail. Our work revealed that addition of exogenous PSY1 leads to transcription of cell wall modifying enzymes, enzymes that might contribute to the loosening of the cell wall during elongation. Two parallel responses to PSY1 were found to exist (PSY1R-dependent and PSY1R-independent). This could suggest that other receptors for PSY1 peptides exist within the plant. PSY1-responsive genes encode several genes localized in cell wall that regulate carboxylestrase activity, while differentially expressed genes in psy1r mutant plants largely were localized to the nucleus with molecular function of ion binding and transcription factor activity. A major part of PSY1responsive genes were involved in cell growth, cell differentiation and catabolic processes. Genome wide gene expression profiling based on GO revealed that most differentially expressed genes were involved in cellular functions and response to stimuli. This suggests a crosstalk between developmental cues and environmental stimuli. A promoter analysis revealed a specific ciselement present in $9 \%$ of the differentially expressed genes of psy1r mutant plants. This element has previously been found in genes regulated by light. Elongation growth of hypocotyls is closely linked to light and one can speculate that PSY1R is involved in the regulation of light response.

\section{Methods}

\section{Plant material and growth conditions}

Experiments with Arabidopsis thaliana were performed on ecotype Columbia- 0 . Twenty-five milligram $(\mathrm{mg})$ of seeds of two plant lines (wild type and psy1r knockout) were surface sterilized in a micro-centrifuge tube by treating them with ethanol and subsequently Klorin containing $0.2 \%$ tween for $10 \mathrm{~min}$. Seeds were then washed again with ethanol for $10 \mathrm{~min}$ and rinsed with sterile water twice before subjecting them to imbibition and stratification at $4^{\circ} \mathrm{C}$ for three days. After stratification, seeds of the two plant lines were grown hydroponically.

Nutrient solution of hydroponic culture containing half strength of Murashige and Skoog medium (MS) with $1 \%(\mathrm{w} / \mathrm{v})$ sucrose was prepared $(\mathrm{pH} 5.7, \mathrm{KOH})$ and sterilized by autoclaving. The stratified seeds of the two 
Table 1 Putative cis-regulatory motives statistically over-represented in the promoters of differentially expressed genes

\begin{tabular}{|c|c|c|c|c|c|c|c|c|c|c|c|c|}
\hline \multirow[t]{2}{*}{ Motif } & \multicolumn{4}{|c|}{$\begin{array}{l}\text { PSY1 responsive genes } \\
\text { in wild type ( } 77 \text { genes) }\end{array}$} & \multicolumn{4}{|c|}{$\begin{array}{l}\text { Differentially expressed genes in } \\
\text { psy1r mutant plants ( } 154 \text { genes) }\end{array}$} & \multicolumn{4}{|c|}{$\begin{array}{l}\text { Differentially expressed genes in PSY1 treated wild type plants } \\
\text { compared to PSY treated psy1r mutant plants ( } 261 \text { genes) }\end{array}$} \\
\hline & Hits out of 77 genes & $\%$ & Z-score & $p$-value & Hits out of 154 genes & $\%$ & Z-score & $\mathrm{p}$-value & Hits out of 261 genes & $\%$ & Z-score & $\mathrm{p}$-value \\
\hline TATATA & 58 & 75 & 2.1 & 0.05 & 141 & 92 & 5.7 & 0.001 & 221 & 85 & 3.6 & 0.001 \\
\hline AAAATA & 75 & 97 & 2.0 & 0.05 & 149 & 97 & 3.6 & 0.001 & 256 & 98 & 3.5 & 0.001 \\
\hline AAAATT & 74 & 96 & 2.0 & 0.05 & 148 & 96 & 1.4 & ns & 254 & 97 & 2.6 & 0.005 \\
\hline AATAAA & 76 & 99 & 1.7 & 0.05 & 148 & 96 & 3.1 & 0.001 & 253 & 97 & 3.8 & 0.001 \\
\hline TGTATA & 53 & 68 & 1.7 & 0.05 & 114 & 74 & 3.0 & 0.001 & 199 & 76 & 3.2 & 0.001 \\
\hline TGTATATAT & 2 & 2 & ns & ns & 14 & 9 & ns & ns & 21 & 8 & ns & ns \\
\hline
\end{tabular}

These sequences have been obtained using the programs Promomer (http://bar.utoronto.ca/ntools/cgi-bin/BAR_Promomer.cgi) and Motif Analysis (TAIR). Z-scores and p-values are those calculated by the corresponding program (Promomer or Motif Analysis). 

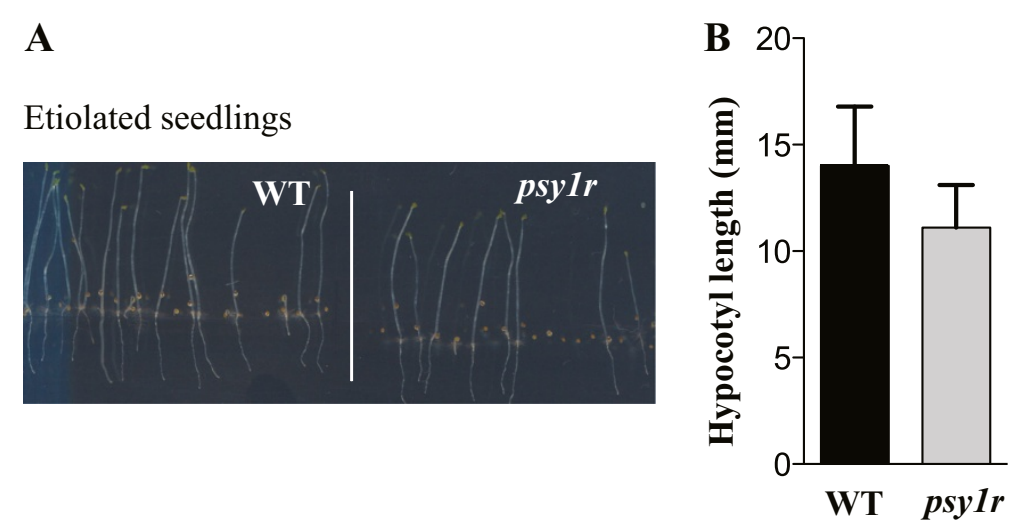

Figure 7 Wild type (WT) and psy1r mutant seedlings grown for $\mathbf{5}$ days in the dark. A) Seeds of wild type and psy $1 r$ mutant plants were grown $1 / 2$ MS $+1 \%$ sucrose agar plates. Results are average $(( \pm$ SE) of minimum of 92 hypocotyls $($ Wt $n=108, p s y 1 r n=92$ analyzed per genotype $(P<0.001$ ANOVA, Tukey test). B) Hypocotyl length $(\mathrm{mm})$ of representative WT and psy1r mutant plant grown in dark.

plant lines were grown in $500 \mathrm{~mL}$-conical flasks containing $250 \mathrm{~mL}$ nutrient solution. Flasks were placed in a growth chamber on a shaker at 180 rpm under continuous light and sterilized conditions. The purified, natural PSY1 was obtained from Yoshikatsu Matsubyashi Lab, National Institute for basic biology, Japan and PSY1 applied to seedlings as described by Amano et al [16]. After one week, plants were treated with the PSY1 peptide at $10 \mathrm{nM}$ concentration for $4 \mathrm{hrs}$ and then transferred into liquid nitrogen and stored at $-80^{\circ} \mathrm{C}$ until RNA extraction.

\section{RNA extraction and microarray}

Total RNA was extracted from three biological replicates of $25 \mathrm{mg}$ seedlings grown in hydroponic culture in sterile conditions using the RNeasy Plant mini kit (Qiagen). RNA integrity was assessed using an Agilent 2100 Bioanalyser with RNA 6000 Nano Assay (Agilent Technologies) and was processed for use on Arabidopsis (V4) Gene Expression Microarray (Agilent Technologies). Arabidopsis (V4) Gene Expression Microarray was used for RNA analysis according to manufacturer's detail (Design ID: 21169, G2519F; Agilent Technologies, Palo Alto, CA, USA). Briefly, $200 \mathrm{ng}$ of total RNA containing RNA spiked in Mix was reverse transcribed in to cDNA that was then in vitro transcribed into cRNA, labeled with cyanine 3-CTP using Agilent Low RNA Input Linear Amp Kit (Agilent Technologies). The Agilent RNA spike-in control targets are a set of 10 in vitro-synthesized polyadenylated transcripts derived from the adenovirus E1A gene used to monitor the labeling reactions and the microarray performance. For labeled cRNA purification, $84 \mu \mathrm{l}$ sterile $\mathrm{H}_{2} \mathrm{O}, 350 \mu \mathrm{l}$ RLT buffer from Qiagen RNAeasy Mini Kit (Qiagen Technologies), and 250- $\mu$ l EtOH were added. The purification steps followed the protocol described by the manufacturer.

After obtaining the required cRNA yield and incorporation rate of fluorescent dye cyanine 3-CTP, a hybridization step was carried out simultaneously for all three biological repeats. Hybridizations were carried out in Agilent's SuperHyb Hybridization Chambers (Agilent Technologies) containing $5 \mu \mathrm{g}$ of cyanine 3-labeled linearly amplified cRNA. The hybridization reaction was performed at $65^{\circ} \mathrm{C}$ for 17 hours using the Agilent DNA microarray hybridization oven (Agilent Technologies), following procedures described in the Agilent One-Color MicroarrayBased Gene Expression Analysis protocol. The hybridized microarrays were disassembled in Agilent Gene Expression Wash Buffer 1 (Agilent Technologies) and then washed with the same buffer for $1 \mathrm{~min}$ at room temperature, followed by washing with Gene Expression Wash Buffer 2 for one min at $37^{\circ} \mathrm{C}$. The microarrays were then scanned immediately using the Agilent DNA Microarray Scanner (Agilent Technologies). The images generated were analyzed with Agilent Feature Extraction Software. The raw data of hybridization was imported into the microarray analysis software GeneSpring 11.5 (Agilent Technologies). Normalization and background intensity determination for each feature performed using the Robust Multiarray Average summarization algorithm, as described by Irizarry et al. [37]. Genes were considered differentially regulated if their normalized expression value was significantly different from the control $(P<0.05)$. One-way ANOVA with Benjamini Hochberg multiple testing corrections (false discovery rate of 0.05) was used to identify genes differentially regulated between treatment groups. Genes exhibiting more than a 2 -fold enhanced or reduced transcription level in three independent experiments were considered to show significant alterations in expression.

\section{Real time PCR}

Aliquots of RNA samples used for the microarray analysis were also analyzed by real-time RT-PCR. Reverse transcription (RT) was performed with $2 \mu \mathrm{g}$ of total 
RNA to obtain cDNA with SuperScript II and Oligo (dT)12-18 (Invitrogen) as the primer in a $20 \mu \mathrm{l}$ reaction volume. Each cDNA sample was diluted 1:4 in sterile $\mathrm{ddH} 2 \mathrm{O}$, and $1 \mu \mathrm{l}$ of this dilution was used as template for qPCR. Primers for the PCR reactions were designed by Beacon Designer ${ }^{\mathrm{rm}}$ to have a Tm of $\sim 60^{\circ} \mathrm{C}$ and an optimal annealing temperature of $53-55^{\circ} \mathrm{C}$ with the length of the amplicons between 120 and 300 bp. Real-time PCR was performed with DyNAmo ${ }^{\text {ma }}$ Flash SYBR ${ }^{\circ}$ Green qPCR Kit (Qiagen) in $20 \mu \mathrm{L}$ reactions according to manufacturer's instruction. Each PCR reaction contains $5 \mu \mathrm{l}$ of diluted cDNA (100 ng), $5 \mu \mathrm{l}(0.5 \mu \mathrm{M})$ of both primers and $10 \mu \mathrm{l}$ of DyNAmo ${ }^{\text {ta }}$ Flash SYBR $^{\circ}$ Green master mix. The initial denaturing time was $7 \mathrm{~min}$ at $95^{\circ} \mathrm{C}$, followed by 45 cycles consisting of $95^{\circ} \mathrm{C}$ for $10 \mathrm{~s}, 57^{\circ} \mathrm{C}$ for $15 \mathrm{~s}, 68^{\circ} \mathrm{C}$ for $30 \mathrm{~s}$ and $75^{\circ} \mathrm{C}$ for $1 \mathrm{~s}$ with a single fluorescence measurement. Then it was held at $60^{\circ} \mathrm{C}$ for $60 \mathrm{~s}$. A melting curve analysis of the generated products $\left(65^{\circ} \mathrm{C}-95^{\circ} \mathrm{C}\right.$ with a heating rate of $1^{\circ} \mathrm{C} \mathrm{s}^{-1}$ and a continuous fluorescence measurement) was performed after the PCR cycles.

ACT2 (AT3G18780) was selected as a valid housekeeping gene since the expression of ACT2 did not change significantly in plant lines treated with the PSY1 peptide compared to untreated plant lines (Additional file 6: Figure S1). In addition to this, no significant changes in expression of $A C T 2$ could be observed in the microarray data further demonstrating that the $A C T 2$ expression is unaffected by the PSY1 peptide treatment. For relative quantification, amplification efficiencies (E) for primer set targeting each gene were determined in the following way: an aliquot of cDNA transcribed from $5 \mu \mathrm{g}$ of total RNA was diluted with sterile $\mathrm{ddH}_{2} \mathrm{O}$ to $10^{-1}, 10^{-2}$ and $10^{-3}$. Standard curves for each gene were performed using the undiluted and diluted cDNA to cover the range of all template concentrations. The specific primers for each gene were used. Gene-specific PCR efficiency was used to calculate the expression of target genes relative to the expression of $A C T 2$ reference gene. The $\triangle C T$ value was calculated as follows: $\triangle \mathrm{CT}$ (target genes) $=\mathrm{CT}$ (target gene) $-\mathrm{CT}$ (Reference gene), where $\mathrm{CT}$ is the cycle number at which PCR product exceeded a set threshold. Relative transcript level (RTL) was calculated through $=1 \times 2^{-\Delta \mathrm{CT}}$.

\section{Gene ontology}

Gene ontologies were analyzed for term enrichment using the AgriGO Single Enrichment Analysis tool with TAIR10 GO annotation (http://bioinfo.cau.edu.cn/agriGO/). GO enrichment was performed in AgriGO (FDR correction and Fisher's exact test $<0.05$ ) using the whole Arabidopsis genome as the background/reference.

Hypocotyl length measurements in dark grown plants Seedlings of psy1r and wild type plants were grown on MS medium $(0.8 \%(\mathrm{w} / \mathrm{v})$ agar and $1 \%(\mathrm{w} / \mathrm{v})$ sucrose $)$ at $22^{\circ} \mathrm{C}$ for 5 days. Seedlings were transferred to transparencies, scanned and measured using the application ImageJ for hypocotyl length measurements.

\section{Availability of supporting data}

The data sets supporting the results of this article are included within the article (and its additional files along with list of genes). Raw microarray data were deposited to GEO public database and available under the accession number "GSE55684". (http://www.ncbi.nlm.nih.gov/ geo/query/acc.cgi?acc=GSE55684).

\section{Additional files}

\begin{abstract}
Additional file 1: Table S1. List of genes that were differentially expressed in wild type plants after PSY1 treatment. Genes were identified using the criteria; $P<0.05$ and fold change $>2$ or $<-2$ through One-way ANOVA (with Benjamini Hochberg multiple testing corrections and FDR < 0.05) between control and peptide PSY1 treated wild type plants. The up-regulated and down regulated genes were sorted from highest to lowest fold expression values.
\end{abstract}

Additional file 2: Table S2. List of all genes differentially expressed in psy $1 r$ mutant plant compared to wild type plants. Genes were identified using the criteria; $\mathrm{P}<0.05$ and fold change $>2$ or $<-2$ through One-way ANOVA (with Benjamini Hochberg multiple testing corrections and FDR $<0.05$ ) between psy $1 r$ mutant plants and wild type plants. The up-regulated and down regulated genes were sorted from highest to lowest fold expression values.

Additional file 3: Table S3. List of genes differentially expressed in PSY1-treated psy1r mutant plants compared to peptide treated wild type plants. Genes were identified using the criteria; $P<0.05$ and fold change $>2$ or $<-2$ through One-way ANOVA (with Benjamini Hochberg multiple testing corrections and FDR $<0.05$ ) between PSY1 treated psy $1 r$ mutant plants and PSY1 treated-wild type plants. The up-regulated and down regulated genes were sorted from highest to lowest fold expression values.

Additional file 4: Table S4. Functional grouping of genes showing differential transcript expression in different treatments. Gene ontology (GO) enrichment was performed in AgriGO (FDR correction and Fisher's exact test $<0.05$ ) using the whole Arabidopsis genome as the background/reference.

Additional file 5: Table S5. Primers used for RT-PCR validation of selected genes identified by microarray after PSY1 treatment in wild type plants.

Additional file 6: Figure S1. The ACT2 (At3g18780) gene expression. The expression of ACT2 was not significantly altered in both plant lines treated with the PSY1 peptide compared to the untreated plant lines. The significance level was tested among three independent biological replicates $(n=3)$. The scale bars represent standard error (S.E) among three biological samples.

\section{Abbreviation}

PSY1: Plant peptide containing sulfated tyrosine 1; PSY1R: Receptor of plant peptide containing sulfated tyrosine 1; RNA: Ribonucleic acid; LRR-LK: Leucine rich repeat receptor like kinase; GO: Gene Ontology; RT-PCR: Real time polymerase chain reaction; WVD2: Wave dampened2; BP: Biological processes; CC: Cellular component; MF: Molecular function; ABA: Abscisic acid; MS: Murashige and Skoog medium; RT: Reverse transcription; RTL: Relative transcript level; SEA: Singular enrichment analysis; ANOVA: Analysis of variance.

\section{Competing interests}

The authors declare they have no competing interests. 


\section{Author's contribution}

$\mathrm{KM}, \mathrm{KJ}$ and ATF designed the study. KM prepared the material. KJ and RK helped in conducting RT-PCR and microarray experiments. KM, KJ, and ATF, analyzed microarray data and identify common motif in promoter region of differentially expressed regions. KM, KJ and ATF wrote the manuscript. ATF and $\mathrm{KJ}$ supervised the project. All authors read and approved the final manuscript.

\section{Acknowledgements}

The projected was supported by a grant from the Danish Research Foundation, FNU, \#272-08-0504 (to ATF) and by the PUMPkin Centre - a Centre of Excellence funded by the Danish National Research Foundation.

Received: 20 December 2013 Accepted: 20 May 2014

Published: 6 June 2014

\section{References}

1. Sparks E, Wachsman G, Benfey PN: Spatiotemporal signalling in plant development. Nat Rev Genet 2013, 14(9):631-644.

2. Murphy E, Smith S, De Smet I: Small signaling peptides in Arabidopsis development: how cells communicate over a short distance. Plant $\mathrm{Cell}$ 2012, 24(8):3198-3217.

3. Wang YH, Irving HR: Developing a model of plant hormone interactions. Plant Signal Behav 2011, 6(4):494-500

4. Zan Y, Ji Y, Zhang Y, Yang S, Song Y, Wang J: Genome-wide identification, characterization and expression analysis of populus leucine-rich repeat receptor-like protein kinase genes. BMC Genomics 2013, 14:318.

5. Walker JC, Zhang R: Relationship of a putative receptor protein kinase from maize to the S-locus glycoproteins of Brassica. Nature 1990, 345(6277):743-746

6. Lee JS, Torii KU: A tale of two systems: peptide ligand-receptor pairs in plant development. Cold Spring Harb Symp Quant Biol 2012, 77:83-89.

7. Matsubayashi Y: Ligand-receptor pairs in plant peptide signaling. J Cell Sci 2003, 116:3863-3870.

8. Kholodenko BN: Cell-signalling dynamics in time and space. Nat Rev Mol Cell Biol 2006, 7(3):165-176

9. Xiong L, Schumaker KS, Zhu JK: Cell signaling during cold, drought, and salt stress. Plant Cell 2002, 14(Suppl):S165-S183.

10. Chow B, McCourt P: Plant hormone receptors: perception is everything. Genes Dev 2006, 20(15):1998-2008.

11. Harashima $\mathrm{H}$, Schnittger $\mathrm{A}$ : The integration of cell division, growth and differentiation. Curr Opin Plant Biol 2010, 13(1):66-74

12. Gray WM: Hormonal regulation of plant growth and development. PLOS Biol 2004, 2(9):E311.

13. Mitchell CA, Myers PN: Mechanical stress regulation of plant growth and development. Hortic Rev 1995, 17:1-42

14. Montgomery BL: Right place, right time: Spatiotemporal light regulation of plant growth and development. Plant Signal Behav 2008, 3(12):1053-1060.

15. Czyzewicz N, Yue K, Beeckman T, Smet ID: Message in a bottle: small signalling peptide outputs during growth and development. J Exp Bot 2013, 64(17):5281-5296.

16. Amano $Y$, Tsubouchi H, Shinohara H, Ogawa M, Matsubayashi Y. Tyrosine-sulfated glycopeptide involved in cellular proliferation and expansion in Arabidopsis. Proc Natl Acad Sci U S A 2007, 104(46):18333-18338.

17. Mosher S, Kemmerling B: PSKR1 and PSY1R-mediated regulation of plant defense responses. Plant Signal Behav 2013, 8(5):e24119.

18. Mosher S, Seybold H, Rodriguez P, Stahl M, Davies KA, Dayaratne S, Morillo SA Wierzba M, Favery B, Keller H, Tax FE, Kemmerling B: The tyrosine-sulfated peptide receptors PSKR1 and PSY1R modify the immunity of Arabidopsis to biotrophic and necrotrophic pathogens in an antagonistic manner. Plant $\rfloor$ 2013, 73(3):469-482.

19. Du Z, Zhou X, Ling Y, Zhang Z, Su Z: agriGO: a GO analysis toolkit for the agricultural community. Nucleic Acids Res 2010, 38:64-70.

20. Muller K, Linkies A, Vreeburg RA, Fry SC, Krieger-Liszkay A, Leubner-Metzger G In vivo cell wall loosening by hydroxyl radicals during cress seed germination and elongation growth. Plant Physiol 2009, 150(4):1855-1865.

21. Willats WG, Orfila C, Limberg G, Buchholt HC, van Alebeek GJ, Voragen AG, Marcus SE, Christensen TM, Mikkelsen JD, Murray BS, Knox JP: Modulation of the degree and pattern of methyl-esterification of pectic homogalacturonan in plant cell walls. Implications for pectin methyl esterase action, matrix properties, and cell adhesion. J Biol Chem 2001, 276(22):19404-19413.
22. Yu L, Sun J, Li L: PtrCel9A6, an Endo-1,4-beta-Glucanase, Is Required for Cell Wall Formation during Xylem Differentiation in Populus. Mol Plant 2013, 6(6):1904-1917.

23. Sato S, Kato T, Kakegawa K, Ishii T, Liu YG, Awano T, Takabe K, Nishiyama Y, Kuga S, Nakamura Y, Tabata S, Shibata D: Role of the putative membrane-bound endo-1,4-beta-glucanase KORRIGAN in cell elongation and cellulose synthesis in Arabidopsis thaliana. Plant Cell Physiol 2001, 42(3):251-263.

24. Hayashi T: The primary wall controls plant cell growth. Tanpakushitsu kakusan koso Protein, nucleic acid, enzyme 1992, 37(15):2968-2976.

25. Karin M: Signal transduction and gene control. Curr Opin Cell Biol 1991, 3(3):467-473.

26. Giehl RF, Lima JE, von Wiren N: Localized iron supply triggers lateral root elongation in Arabidopsis by altering the AUX1-mediated auxin distribution. Plant Cell 2012, 24(1):33-49.

27. Sancenon V, Puig S, Mateu-Andres I, Dorcey E, Thiele DJ, Penarrubia L: The Arabidopsis copper transporter COPT1 functions in root elongation and pollen development. J Biol Chem 2004, 279(15):15348-15355.

28. Xin Z, Wang A, Yang G, Gao P, Zheng ZL: The Arabidopsis A4 subfamily of lectin receptor kinases negatively regulates abscisic acid response in seed germination. Plant Physiol 2009, 149(1):434-444.

29. Fan J, Hill L, Crooks C, Doerner P, Lamb C: Abscisic Acid Has a Key Role in Modulating Diverse Plant-Pathogen Interactions. Plant Physiol 2009, 150(4):1750-1761.

30. Chandler J, Wilson A, Dean C: Arabidopsis mutants showing an altered response to vernalization. Plant J 1996, 10(4):637-644

31. Gremski K, Ditta G, Yanofsky MF: The HECATE genes regulate female reproductive tract development in Arabidopsis thaliana. Development 2007, 134(20):3593-3601

32. Alvarez JP, Goldshmidt A, Efroni I, Bowman JL, Eshed Y: The NGATHA distal organ development genes are essential for style specification in Arabidopsis. Plant Cell 2009, 21(5):1373-1393.

33. Toufighi K, Brady SM, Austin R, Ly E, Provart NJ: The Botany Array Resource: e-Northerns, Expression Angling, and promoter analyses. Plant J 2005, 43(1):153-163.

34. Hobson GM, Mitchell MT, Molloy GR, Pearson ML, Benfield PA: Identification of a novel TA-rich DNA binding protein that recognizes a TATA sequence within the brain creatine kinase promoter. Nucleic Acids Res 1988, 16(18):8925-8944.

35. Hudson ME, Quail PH: Identification of promoter motifs involved in the network of phytochrome A-regulated gene expression by combined analysis of genomic sequence and microarray data. Plant Physiol 2003, 133(4):1605-1616.

36. Lee YK, Kim GT, Kim IJ, Park J, Kwak SS, Choi G, Chung WI: LONGIFOLIA1 and LONGIFOLIA2, two homologous genes, regulate longitudinal cell elongation in Arabidopsis. Development 2006, 133(21):4305-4314.

37. Irizarry RA, Hobbs B, Collin F, Beazer-Barclay YD, Antonellis KJ, Scherf U, Speed TP: Exploration, normalization, and summaries of high density oligonucleotide array probe level data. Biostatistics 2003, 4(2):249-264.

doi:10.1186/1471-2164-15-44

Cite this article as: Mahmood et al:: Analysis of peptide PSY1 responding transcripts in the two Arabidopsis plant lines: wild type and psy1r receptor mutant. BMC Genomics 2014 15:441.

\section{Submit your next manuscript to BioMed Central and take full advantage of:}

- Convenient online submission

- Thorough peer review

- No space constraints or color figure charges

- Immediate publication on acceptance

- Inclusion in PubMed, CAS, Scopus and Google Scholar

- Research which is freely available for redistribution 